\title{
P08-03. Genetic complexity of TRIM5 and APOBEC in East Africa
} AM Walsh ${ }^{5}$, K Perret ${ }^{5}$, R Koehler ${ }^{5}$, N Moqueet ${ }^{5}$, C Bautista ${ }^{5}$, L Eller ${ }^{1}$, M Eller ${ }^{1}$, H Kibuuka ${ }^{1}$, M Hoelscher ${ }^{2}$, L Maboko ${ }^{3}$, F Wabwire-Mangen ${ }^{1}$, ML Robb ${ }^{5}$, NL Michael ${ }^{4}$, F McCutchan ${ }^{5}$, J Kim ${ }^{4}$ and GH Kijak*5

Address: ${ }^{1}$ Makerere University Walter Reed Project, Kampala, Uganda, ${ }^{2}$ Dept. of Infectious Diseases \& Tropical Medicine, Univ. of Munich, Munich, Germany, ${ }^{3}$ Mbeya Medical Research Program, Mbeya, Tanzania, ${ }^{4}$ US MIlitary HIV Research Program/WRAIR, Rockville, MD, USA and ${ }^{5}$ US MIlitary HIV Research Program/Henry M. Jackson Foundation, Rockville, MD, USA

* Corresponding author

from AIDS Vaccine 2009

Paris, France. 19-22 October 2009

Published: 22 October 2009

Retrovirology 2009, 6(Suppl 3):PIII doi:10.II86/I742-4690-6-S3-PIII

This abstract is available from: http://www.retrovirology.com/content/6/S3/PI I I

(C) 2009 Walsh et al; licensee BioMed Central Ltd.

\section{Background}

Host restriction factors (HRFs) TRIM5alpha and APOBECs effect post-entry inhibition of HIV-1 replication in vitro but in vivo significance remains unclear. By acting as a first line of defense, HRFs could potentially act synergistically with adaptive/vaccine-induced immunity. HRFs are polymorphic in world populations, but little is known about their variation in East Africa, despite its high rate of HIV-1 infection. This study describes trim5 and apobec polymorphisms in East African populations.

\section{Methods}

East African HIV-negative adults from Mbeya, Tanzania ( $\mathrm{n}$ $=174)$ and Kampala, Uganda $(\mathrm{n}=160)$ were studied. Putative polymorphic loci in trim5 $(n=10)$, apobec $3 b(n$ $=6)$, apobec $3 f(n=7)$, and apobec $3 g(n=11)$ were genotyped from gDNA using real-time PCR. Genetic analyses were conducted (Pypop/Arlequin/Genepop/Haploview).

\section{Results}

Genotyping data was obtained in 11244/11356 (99.0\%) reactions. Extensive genetic polymorphism was observed: 8/10 trim5 and 14/24 apobec loci exhibited minor allele frequencies (MAFs) >0.05 (range: 0.070-0.475), without significant deviations from Hardy-Weinberg equilibrium. Genic/genotypic differentiation tests failed to reject the null hypothesis that alleles and genotypes were drawn from the same distribution in the two populations. TRIM5alpha 136Q (coiled-coil domain) was represented at frequencies of 0.075 and 0.073 in Uganda and Tanzania, respectively. Variation at TRIM5alpha codon 419 (SPRY domain) was low (MAF<0.01). Genotype frequencies of $186 \mathrm{R} / \mathrm{R}$ in APOBEC3G were $10.8 \%$ in Uganda and $14.8 \%$ in Tanzania. Among apobecs, strong intragenic linkage disequilibrium was observed but was weaker between loci in different genes. No trim 5 or apobec haplotype predominated, and there was congruence in the most common haplotypes in Uganda and Tanzania.

\section{Conclusion}

This initial description of trim5 and apobec in East Africa reveals extensive genetic diversity, with similarity in the distribution of alleles and haplotypes in HRFs among Ugandan and Tanzanian populations, making this a preferred setting to define the impact of HRF variation on HIV acquisition, disease progression, and vaccine efficacy. 